\title{
Role of palliative radiotherapy in unresectable intrahepatic cholangiocarcinoma: population- based analysis with propensity score matching
}

This article was published in the following Dove Press journal:

Cancer Management and Research

\section{Feng Shao* \\ Wei Qi* \\ Fu Tao Meng* \\ Lujun Qiu \\ Qiang Huang}

Department of Biliary-Pancreatic Surgery, Anhui Provincial Hospital Affiliated with Anhui Medical University, Hefei, Anhui, People's Republic of China

*These authors contributed equally to this work
Correspondence: Qiang Huang

Department of Biliary-Pancreatic Surgery, Anhui Provincial Hospital Affiliated with

Anhui Medical University, 19 Lu Jiang

Road, Hefei 23000I, Anhui, People's

Republic of China

$\mathrm{Tel}+8655162283484$

Fax +86 55I 62283325

Email tagsmile1985@I63.com
Background: This population-based study evaluated the overall (OS) and cancer-specific survival (CSS) benefit from palliative radiotherapy (RT) in patients with unresectable intrahepatic cholangiocarcinoma (ICC).

Methods: We queried The Surveillance, Epidemiology, and End Results (SEER) database for the patients with unresectable ICC diagnosed from 1973 to 2013. Propensity score-matched analysis was performed to reduce the impact of the selection bias between the palliative RT group and the nonpalliative RT group. Kaplan-Meier survival curves were used to estimate the survival outcome before and after propensity score matching. OS and CSS were compared between patients with and without palliative RT using univariate and multivariate Cox proportional hazards regression analyses.

Results: A total of 4,027 of 15,803 patients diagnosed with ICC were included in this study. Of those, 847 (21\%) patients underwent palliative RT, whereas 3,180 (79\%) did not. In the unmatched population, patients treated with palliative RT had improved OS and CSS relative to those treated without palliative $\mathrm{RT}$ (adjusted $\mathrm{HR}=0.9065,95 \% \mathrm{CI}=0.8360-0.982, P=0.01735$ ) and CSS (adjusted HR $=0.8874,95 \% \mathrm{CI}=0.8160-0.9652, P=0.00532$ ). After propensity score matching, palliative RT was associated with a significantly improved OS (adjusted HR $=0.8544$, $95 \% \mathrm{CI}=0.7722-0.9453, P=0.00228)$ and $\mathrm{CSS}$ (adjusted HR $=0.8563,95 \% \mathrm{CI}=0.7711-0.9509$, $P=0.0037)$.

Conclusion: Palliative RT seems to improve the prognosis of patients with unresectable ICC. Keywords: radiation therapy, SEER, unresectable intrahepatic cholangiocarcinoma

\section{Background}

Intrahepatic cholangiocarcinoma (ICC) is a relatively rare gastrointestinal malignancy that originates from the epithelial lining of the intrahepatic biliary tree. Despite a rising incidence, existing data to inform optimal treatment are limited due to the rarity of the disease. ${ }^{1-3}$

Radical surgical resection is generally considered the only curative treatment for ICC. However, the majority of patients are unresectable due to advanced disease at the time of diagnosis. ${ }^{4,5}$ For patients with inoperable disease, the overall prognosis remains poor, with the median survival being 7-12 months. ${ }^{6}$ The results of the Advanced Biliary Cancer Trial demonstrated that cisplatin plus gemcitabine treatment can improve the overall survival (OS) in locally advanced and metastatic biliary tumors. ${ }^{7}$ Cisplatin plus gemcitabine treatment is generally considered the standard frontline therapy for locally advanced biliary tumors, which is often followed by transarterial chemoembolization, thermal ablation, and external radiotherapy (RT). 
Previous studies have suggested that RT can improve the OS in patients with unresectable ICC. ${ }^{6,8-10}$ On the other hand, some papers reported that RT has no impact on OS rate of patients with unresectable ICC. ${ }^{11}$ The role of radiation in the management of unresectable ICC remains an important unanswered question. Therefore, we evaluated the OS and cancer-specific survival (CSS) benefit from palliative RT in patients with unresectable ICC.

\section{Methods}

The present study was performed using the Surveillance, Epidemiology, and End Results (SEER) 18-Registry (1973-2013 dataset), which represented approximately $28 \%$ of cancer cases in the USA. This database documented clinical tumor characteristics including diagnosis, race, sex, marital status, tumor-node-metastasis stage, type of cancer and survival time, and cause-of-death information.

The third edition of the International Classification of Diseases for Oncology (ICD-O-3) was used for the identification of patients with ICC. Patients with ICC were selected by ICD-O-3 site codes C22.1 or C22.0. The ICD-O-3 histological codes of 8010, 8020, 8040, 8070, 8041, 8140, 8144, $8160,8161,8162,8163,8260,8310,8480,8490$, and 8560 were used to identify ICC. ${ }^{12}$ We excluded patients without histological diagnostic confirmation or who were diagnosed at autopsy. We also excluded patients with a survival time of $<3$ months, those with other SEER reportable malignancies if ICC was not the first one, those undergoing tumor resection, patients with distant metastases, and those lacking information about RT.

\section{Propensity score matching}

Because of unbalanced baseline characteristics, retrospective studies unavoidably suffer from selection bias. This bias may influence comparison of the survival outcomes between patients treated with and without palliative RT. Propensity score-matching analysis, which is defined as a statistical method to adjust for potential baseline confounding variables, was performed to reduce the impact of the selection bias. A logistic regression model was established for calculating the propensity scores. ${ }^{13}$ Palliative RT status was considered as the dependent variable, while baseline characteristics such as age at diagnosis, gender, race, tumor grade, marital status, tumor size, and cause of death were considered as predefined covariates. The patients who underwent palliative RT were matched with those who did not undergo this procedure on the basis of a greedy algorithm of nearest neighbor matching at a $1: 1$ fixed ratio. ${ }^{14}$

\section{Statistical analysis}

The primary outcome was OS; the secondary outcome was CSS. Categorical patient characteristics were compared using the $\chi^{2}$ test. Kaplan-Meier survival curves were used to estimate the survival outcome before and after propensity score matching. The survival difference according to the receipt of palliative RT was compared using a log-rank test. Cox proportional hazard regression models were used to obtain unadjusted and adjusted HR and $95 \% \mathrm{CI}$ for survival in univariate analysis and multivariate analyses. Sensitivity analysis was performed using random survival forest methodology by repeating computations after substituting unknown data. ${ }^{15}$ Statistical analyses were performed using R3.2.3 for window (http:// www.R-project.org). Propensity score analysis was performed using the MatchIT package. ${ }^{16}$ Random survival forest survival analysis was performed using the "randomForestSRC" package. A 2 -sided $P<0.05$ was considered statistically significant.

\section{Ethics approval and consent to participate}

This study was partly based on the publicly available SEER database, and we received permission of The National Cancer Institute to access the database for the purposes of research only. As this research did not include interaction with humans or use personal identifying information, informed consent was not required.

\section{Results}

\section{Patients' characteristics}

For the present investigation, 4,027 of 15,803 patients diagnosed with ICC were included. Of those, 847 (21\%) patients underwent palliative RT, whereas 3,180 (79\%) did not. Table 1 summarizes clinicopathologic characteristics between the 2 groups. The median age was 64 years (range 20-97). Figure 1 depicts the detailed flow chart of patients' selection for the study cohort.

\section{Survival outcomes in the before matching population}

Figure 2A and B represent Kaplan-Meier curves for OS and CSS survival in patients with and without palliative RT. Patients with palliative RT had a better OS and CSS compared with those not undergoing palliative RT (OS $P=0.0138$, CSS survival $P=0.00453$ ). In univariate analysis, palliative RT was a statistically significant prognostic factor for OS (HR $=0.9024,95 \% \mathrm{CI}=0.832-0.9778, P=0.0122)$ (Table 2) and CSS (HR $=0.885,95 \% \mathrm{CI}=0.814-0.9619, P=0.00406$ ) (Table 3), respectively. In multivariable Cox proportional 
Table I Patient characteristics of the palliative RT and nonpalliative RT groups before and after propensity score matching

\begin{tabular}{|c|c|c|c|c|c|c|}
\hline \multirow[t]{2}{*}{ Variable } & \multicolumn{3}{|c|}{ Patient characteristics in raw data } & \multicolumn{3}{|c|}{ After propensity score matching } \\
\hline & Palliative RT & Nonpalliative RT & $P$-value & Palliative RT & Nonpalliative RT & $P$-value \\
\hline Age, years & & & 0.009 & & & 0.588 \\
\hline$\leq 65$ & 490 & $\mathrm{I}, 679$ & & 490 & 501 & \\
\hline$>65$ & 357 & $\mathrm{I}, 50 \mathrm{I}$ & & 357 & 346 & \\
\hline Gender & & & 0.468 & & & 0.662 \\
\hline Male/female & $433 / 4 \mid 4$ & $\mathrm{I}, 58 \mathrm{I} / \mathrm{I}, 599$ & & $433 / 4 \mid 4$ & $442 / 405$ & \\
\hline Race & & & 0.092 & & & 0.735 \\
\hline White & 688 & 2504 & & 688 & 697 & \\
\hline Black & 49 & 253 & & 49 & 42 & \\
\hline Other/unknown & 110 & 423 & & 110 & 108 & \\
\hline Marital status & & & 0.000 & & & 0.868 \\
\hline Married & 578 & 1,904 & & 578 & 576 & \\
\hline Others & 248 & 1,167 & & 248 & 253 & \\
\hline Unknown & 21 & 109 & & 21 & 18 & \\
\hline Grading & & & 0.103 & & & 0.907 \\
\hline GI & 55 & 204 & & 55 & 56 & \\
\hline G2 & 142 & 577 & & 142 & $|3|$ & \\
\hline G3 & 167 & 514 & & 167 & 167 & \\
\hline G4 & 3 & 24 & & 3 & 5 & \\
\hline Unknown & 480 & $|, 86|$ & & 480 & 488 & \\
\hline Tumor size & & & 0.000 & & & 0.912 \\
\hline$\leq 5 \mathrm{~cm}$ & 177 & 497 & & 177 & 171 & \\
\hline$>5 \mathrm{~cm}$ & 221 & 1,008 & & 221 & 227 & \\
\hline Unknown & 449 & 1,675 & & 449 & 449 & \\
\hline Cause of death & & & 0.290 & & & 0.744 \\
\hline Dead from cancer & 704 & 2,593 & & 704 & 709 & \\
\hline Alive/dead not from cancer & 143 & 587 & & 143 & 138 & \\
\hline
\end{tabular}

Abbreviation: $\mathrm{RT}$, radiotherapy.

analyses, palliative RT was a statistically significant had a significantly protective effect on OS (HR $=0.9065,95 \% \mathrm{CI}$ $=0.8360-0.982, P=0.01735)$ (Table 2$)$ and CSS $(H R=0.8874$, $95 \% \mathrm{CI}=0.8160-0.9652, P=0.00532$ ) (Table 3).

\section{Adjusting for patient characteristics using propensity score matching}

Propensity score-matching analysis was performed to adjust for potential baseline confounding variables including age, marital status, and tumor size. Table 1 shows the details of the variables before and after propensity score matching, noting that the impact of potential selection bias was reduced. The propensity score for patients in the palliative RT group was $0.22 \pm 0.06$ compared with $0.21 \pm 0.05$ for patients in the nonpalliative RT group. After propensity score matching, the score for patients in the palliative RT group was $0.22 \pm 0.06$, and it was $0.22 \pm 0.06$ for patients in the nonpalliative RT group. Figure 3 represents the distribution of the propensity scores of the 2 groups before and after propensity score matching.

Figure 4A and B represent Kaplan-Meier curves for OS and CSS after propensity score matching. Patients with palliative RT had a better OS and CSS compared with those not undergoing palliative RT (OS $P=0.00864$, CSS $P=0.0149)$. In univariate analysis, palliative RT was a statistically significant prognostic factor for OS (HR $=0.8725,95 \% \mathrm{CI}=0.788-0.964$, $P=0.00791$ ) (Table 2) and CSS (HR $=0.8771,95 \% \mathrm{CI}$ $=0.7902-0.9737, P=0.0139$ ) (Table 3$)$ in the matched population. In multivariable Cox analysis, palliative RT was statistically significant and had a significantly protective effect on OS (HR $=0.8544,95 \% \mathrm{CI}=0.7722-0.9453, P=0.00228$ ) (Table 2) and CSS (HR $=0.8563,95 \% \mathrm{CI}=0.7711-0.9509$, $P=0.0037$ ) (Table 3) in the matched population.

\section{Sensitivity analysis}

The overall study results were not affected by the imputation of unknown data using random survival forest methodology. In sensitivity analysis, palliative RT remained a statistically significant protective factor for OS (HR $=0.9114,95 \%$ CI $=0.840-0.989, P=0.02596)$ and $\mathrm{CSS}(\mathrm{HR}=0.8976,95 \% \mathrm{CI}$ $=0.8245-0.9771, P=0.01260)$, respectively.

\section{Discussion}

Our results suggested that palliative RT may improve OS and CSS of patients with unresectable ICC. Both univariate and 


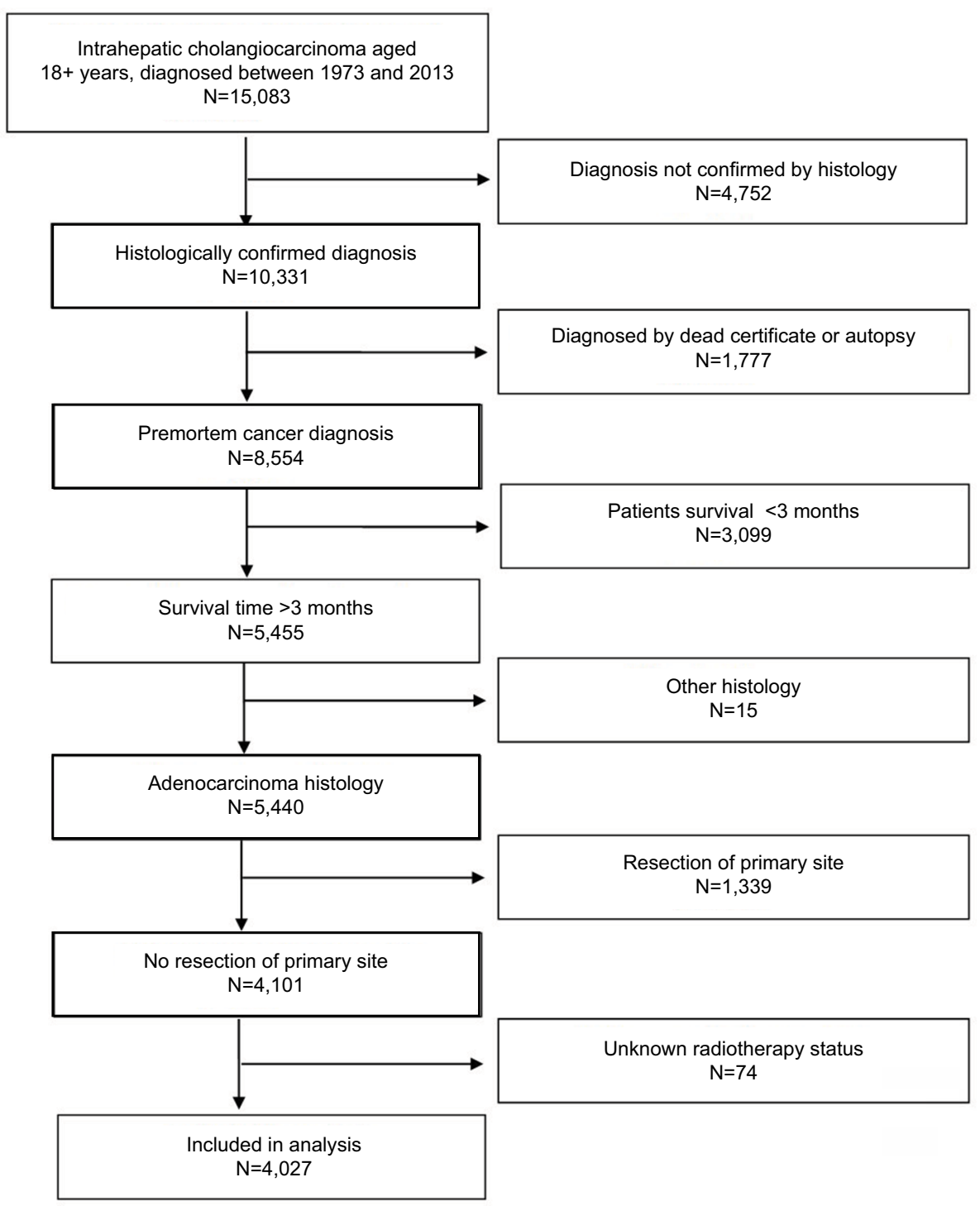

Figure I Flow chart of patients' cohort definition.

multivariable Cox analysis after propensity score matching demonstrated a better prognosis in the patients treated with palliative RT regardless of patient characteristics.

This study is the first analysis based on SEER using propensity score matching to evaluate the effect of palliative RT for unresectable ICC. Similar to our findings, other retrospective studies found palliative RT to have a better prognosis in the patients treated with palliative RT. In a retrospective study series of 84 patients with unresectable ICC, Chen et al ${ }^{9}$ reported a more favorable prognosis treated with external beam RT. In another SEER-based study, Shinohara et $\mathrm{l}^{6}$ reported a significantly reduced overall mortality in the patients treated with palliative RT. There were only 396 patients who received RT. The median survival time was 7 months as against 3 months for patients not treated with RT $(P<0.01)$. The largest study reported an analysis of the
National Cancer Database in 1,636 patients, including 374 patients who received chemotherapy with radiation. ${ }^{10}$ The authors found the survival benefit from the addition of radiation to chemotherapy using the propensity score-matching method. However, the National Cancer Database, which is a hospital-based registry, more likely suffers from selection bias than a population-based study. ${ }^{17}$ Conversely, we used SEER which is a population-based database.

On the other hand, a retrospective study using the nationwide SEER registry found no difference in prognosis between unresectable ICC patients who did undergo palliative RT and those who did not. ${ }^{11}$ However, our results demonstrated a better prognosis in the patients treated with palliative RT. We speculated that this discrepancy might be caused only by selection bias arising from an unbalanced distribution of the baseline characteristics between the 2 groups. 


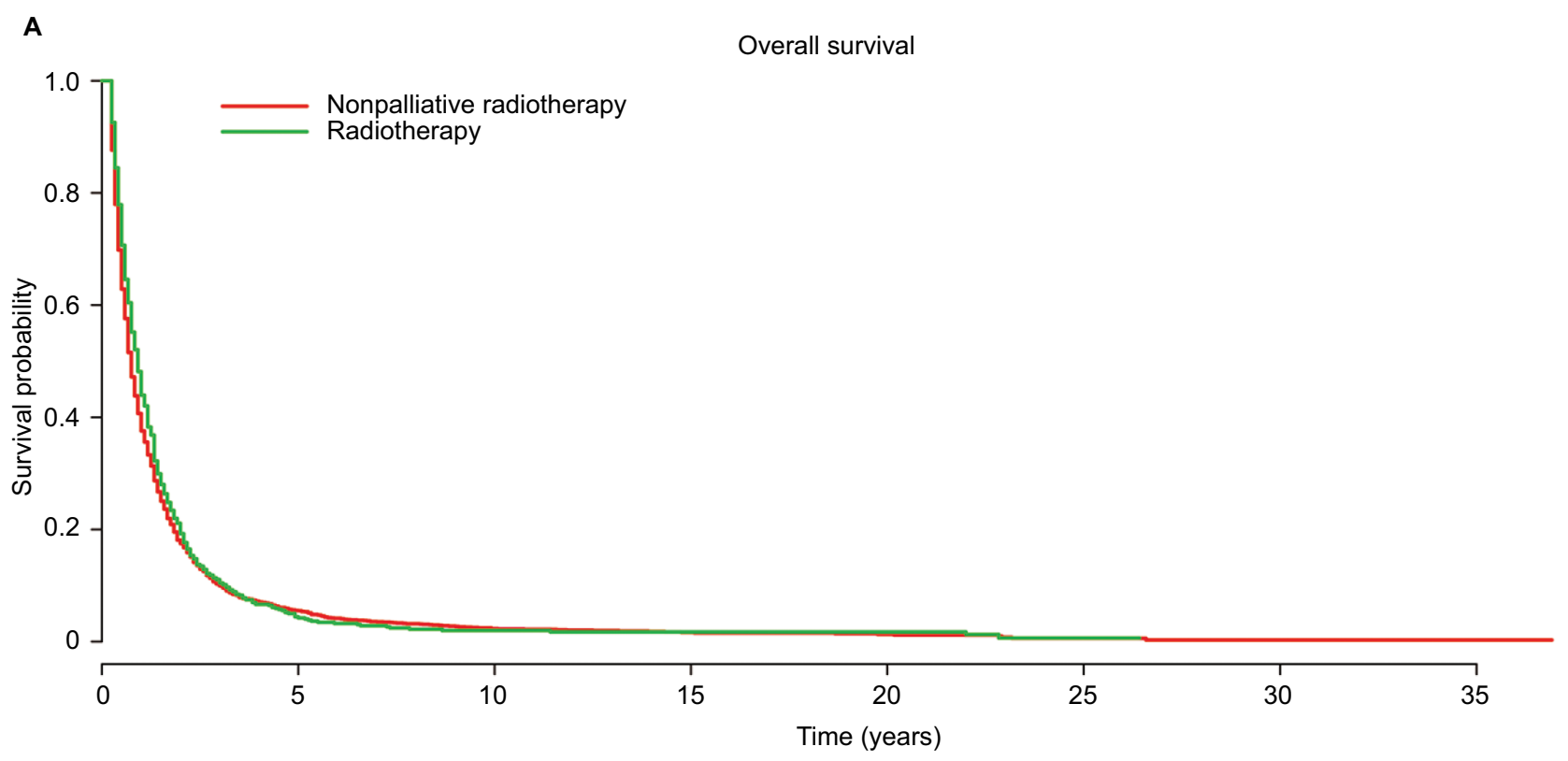

B

Cancer-specific survival

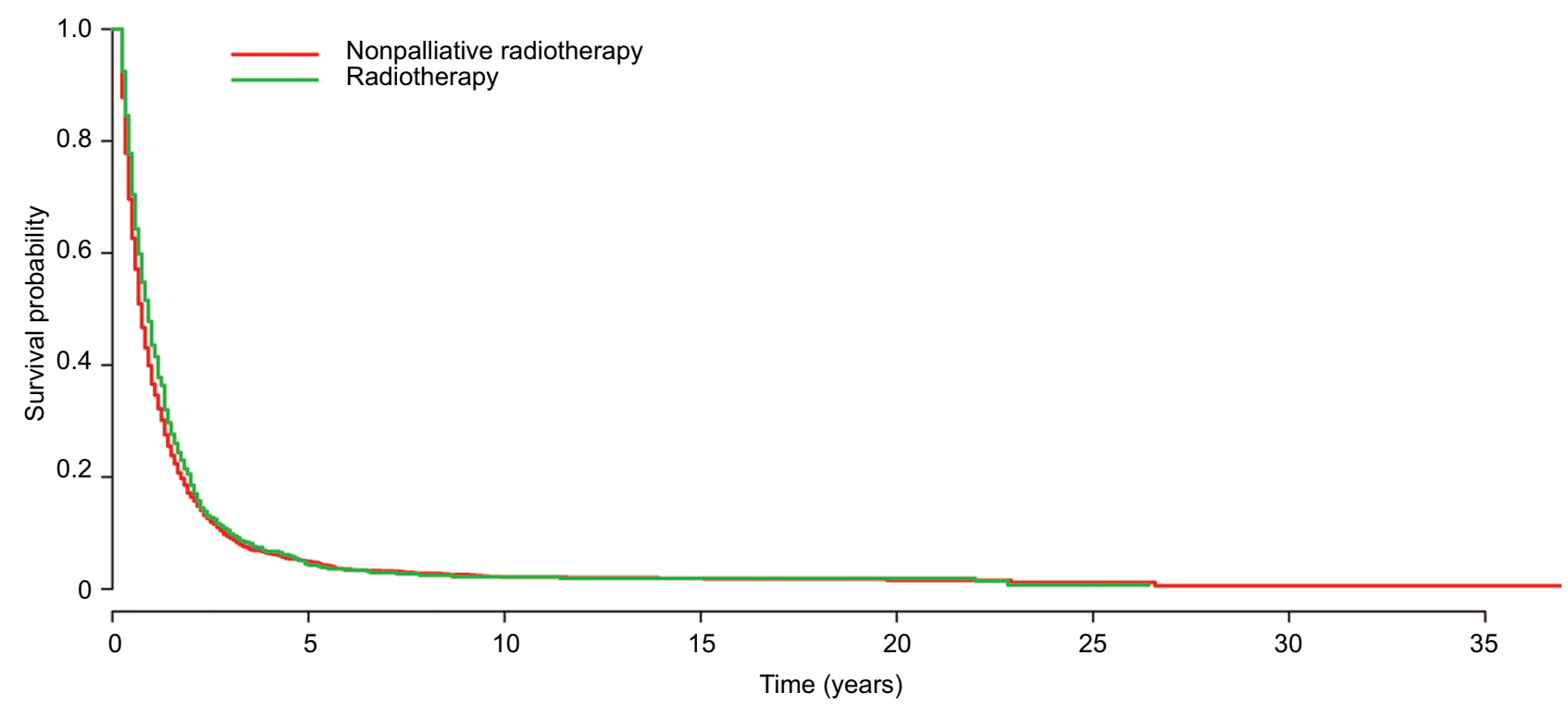

Figure 2 Kaplan-Meier analysis of (A) overall and (B) cancer-specific survival of the palliative radiotherapy and nonpalliative radiotherapy groups before propensity score matching.

The present study is an analysis of SEER data in 4,027 patients, including 847 patients who underwent palliative RT. To the best of our knowledge, this study is the largest report on patients with unresectable ICC who were treated with palliative RT. To minimize the potential selection bias, propensity score matching was carried out in this population-based investigation. By propensity score matching, any imbalance between the 2 groups could be avoided, imbalance that generated effects similar to random allocation. In addition, we performed sen- sitivity analysis by imputation of unknown data using random survival forest methodology. Because our results are consistent, it provides stronger evidence that palliative RT may improve OS and CSS of patients with unresectable ICC.

Many investigators have begun to investigate different strategies of palliative RT for patients with unresectable ICC. Stereotactic body radiation therapy (SBRT) and highdose hypofractionated proton therapy had been reported to be effective and reasonably safe therapies for unresectable 


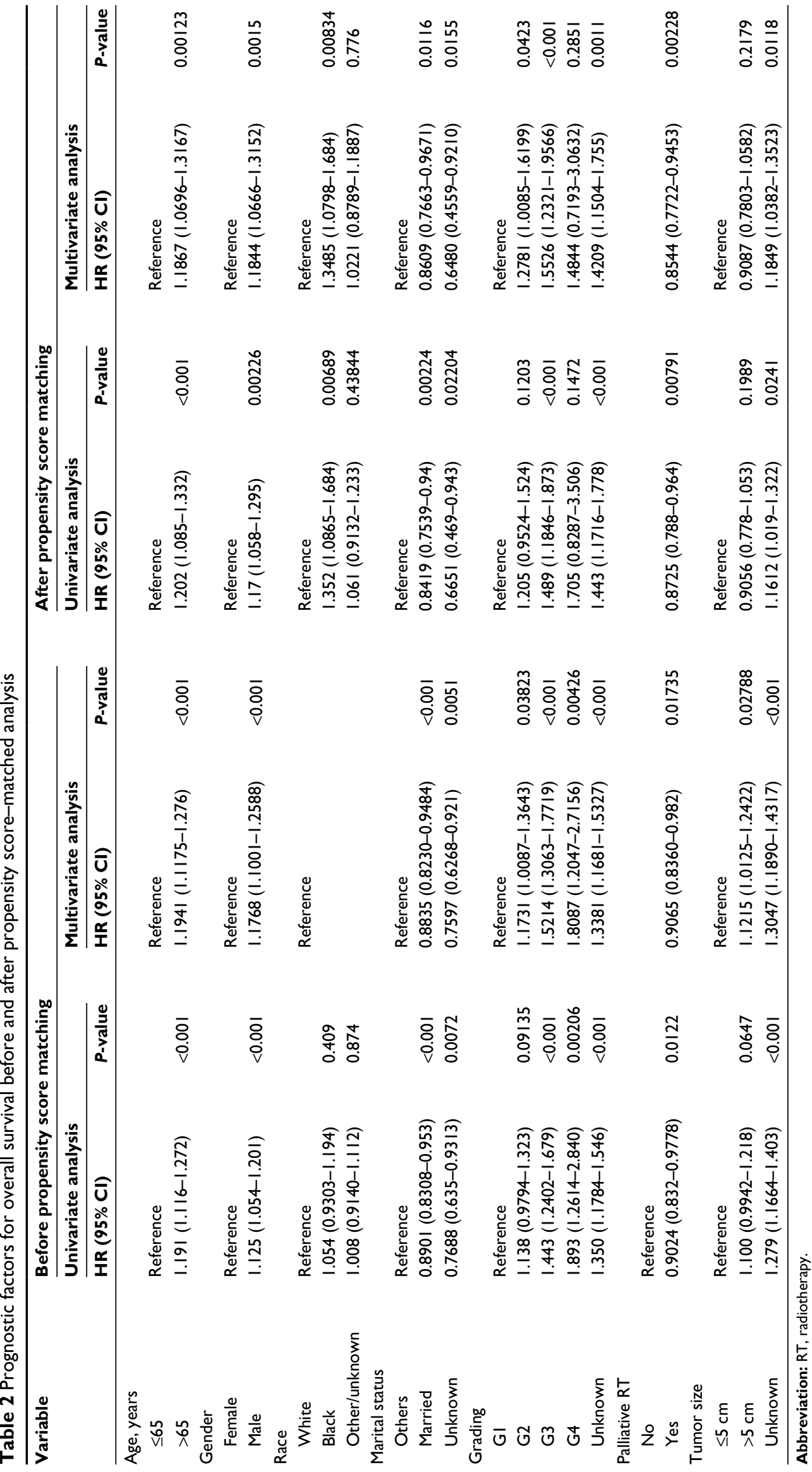




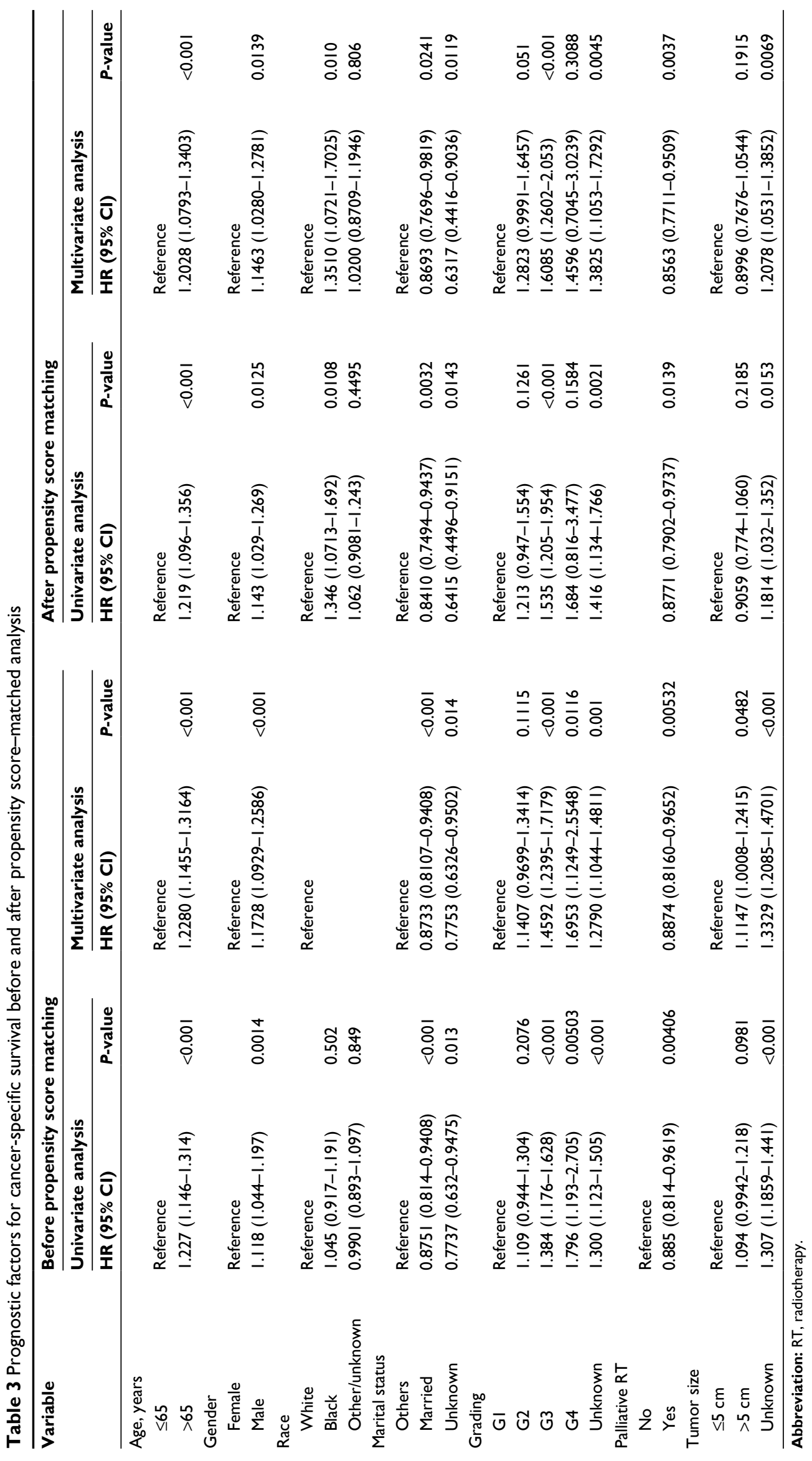



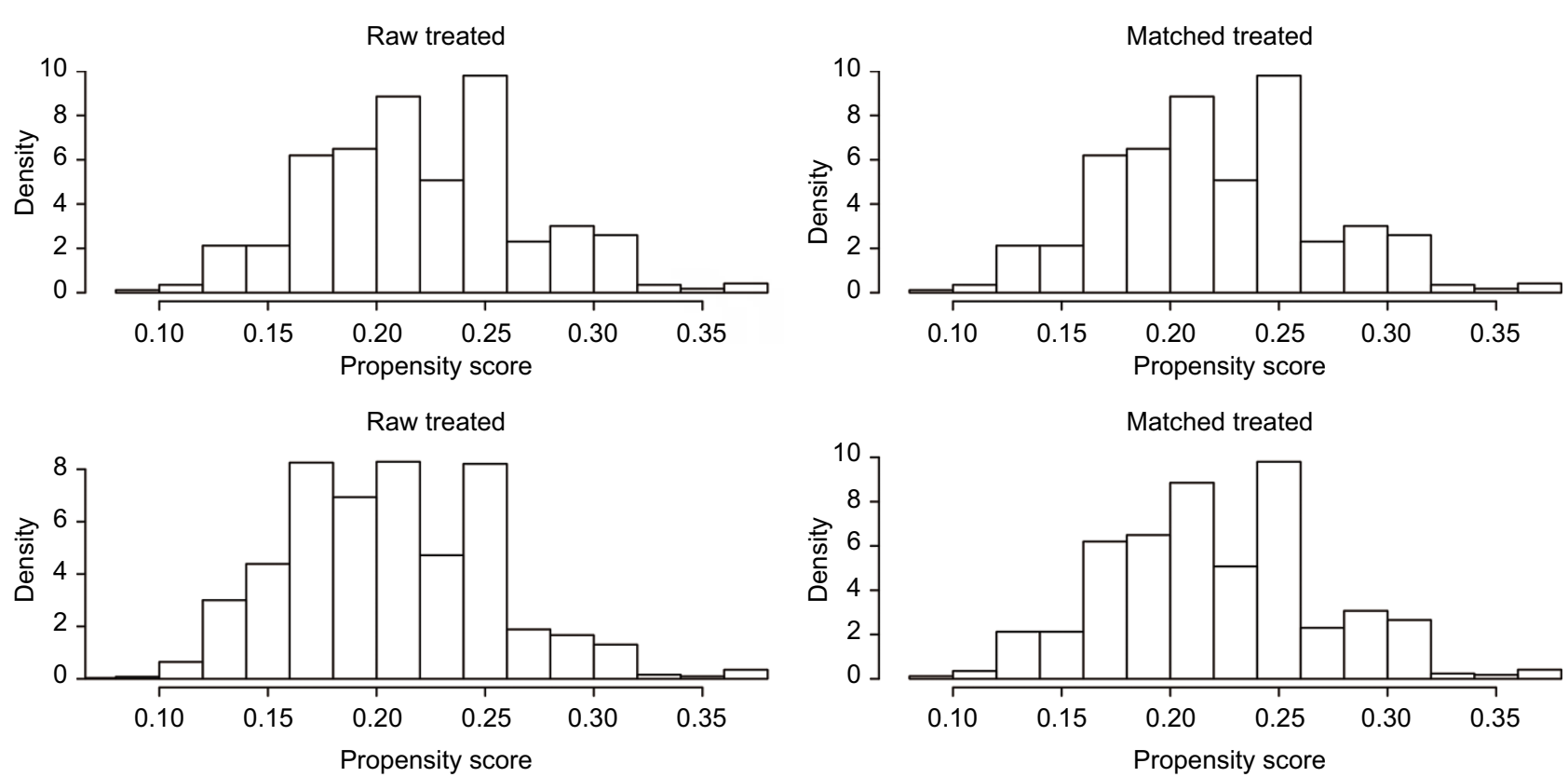

Figure 3 Distribution of propensity score before and after propensity matching procedure.

ICC. ${ }^{18,19}$ Tse et $\mathrm{al}^{20}$ published a Phase I trial of individualized 6-fraction SBRT for unresectable primary liver tumors including 10 patients with unresectable ICC. The median survival of ICC patients was 15 months in their study. Another group also reported a series of 32 patients with unresectable intrahepatic or hilar cholangiocarcinoma, who were treated with SBRT. ${ }^{21}$ The median OS was 17 months and Grade III toxicity was $12 \%$. Thus, the authors concluded that SBRT is an effective and reasonably safe local therapy. More recently, the Massachusetts General Hospital has reported encouraging outcomes using highdose hypofractionated proton therapy. The findings of this multi-institutional Phase II Study were impressive, because the local control rate was $94.8 \%$ and the OS rate at 2 years was $46.5 \% .^{22}$

There are several limitations to the current study that must be acknowledged. First, information on chemotherapy was not available in the SEER registry. Therefore, it is impossible to making any adjustment for the use of chemotherapy. Because chemotherapy was associated with survival, we could not confirm the survival benefit of palliative RT without considering chemotherapy. Moreover, information on physical condition, liver function, and complications, which are important factors influencing patient survival, were not available in the SEER registry. Second, information about palliative RT, such as the dose, fractionation-specific data, and treatment-related toxicity, could not be ascertained in the SEER registry. Third, because the current study is nonrandomized, it unavoidably suffers from selection bias or from the impact of unknown variables that cannot be reduced in the SEER registry. Finally, OS and CSS were the primary and secondary outcomes in this study. Information such as quality of life and local control are important measures, which were unavailable in the SEER registry. Despite these limitations, this is the first large-scale study to evaluate the effect of palliative RT for unresectable ICC. Although some unknown confounders were unadjusted, it appears to be impossible that the large beneficial effects of palliative RT are due solely to unknown confounders. The large sample size and the population-based nature reflect the real-world outcome for patients with unresectable ICC.

\section{Conclusion}

The present study indicates the potential favorable impact of palliative RT on survival outcome in patients with unresectable ICC. Prospective randomized controlled trials are needed to verify the conclusion of this study.

\section{Data sharing statement}

Any request of data and material may be sent to the corresponding author.

\section{Acknowledgment}

The authors acknowledge the efforts of the SEER Program tumor registries in the creation of the SEER database. 
A

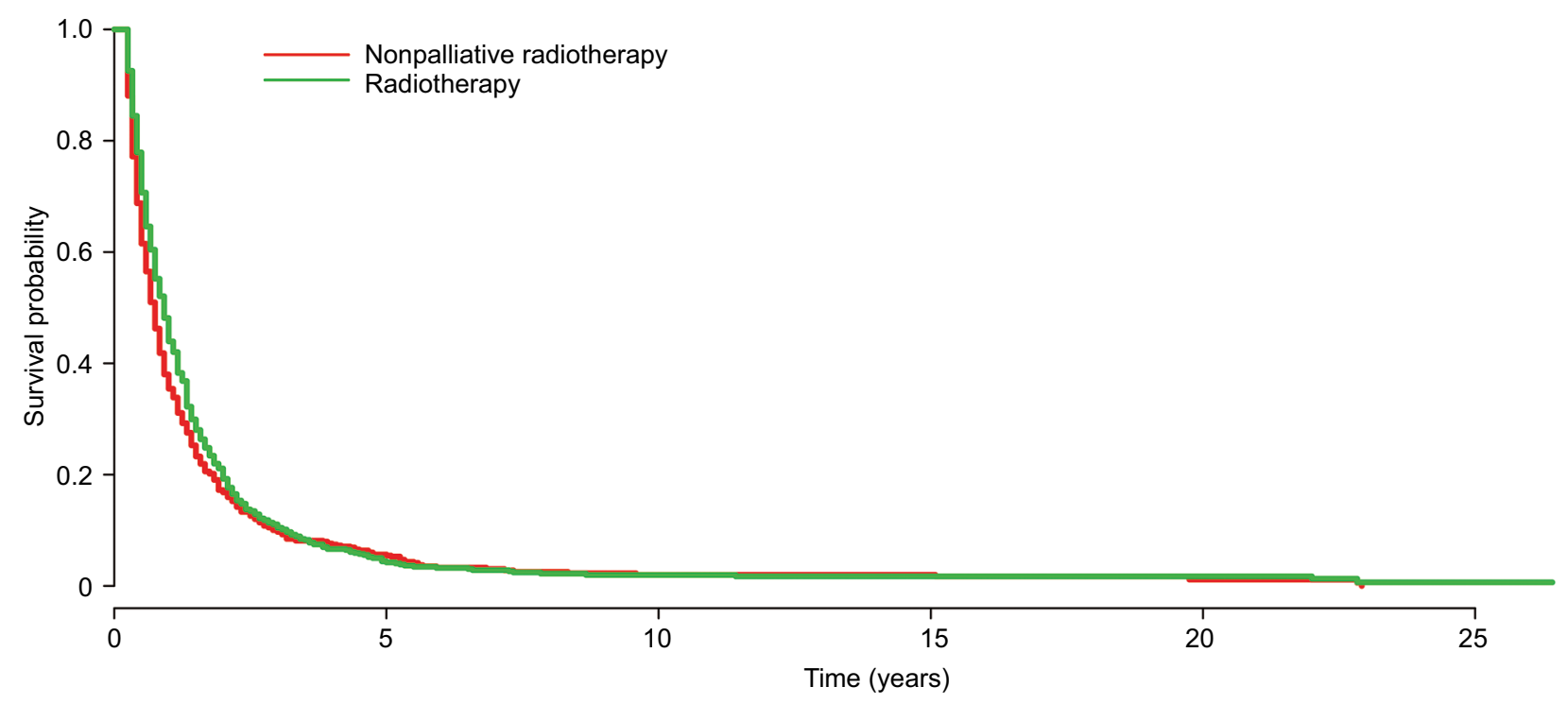

B

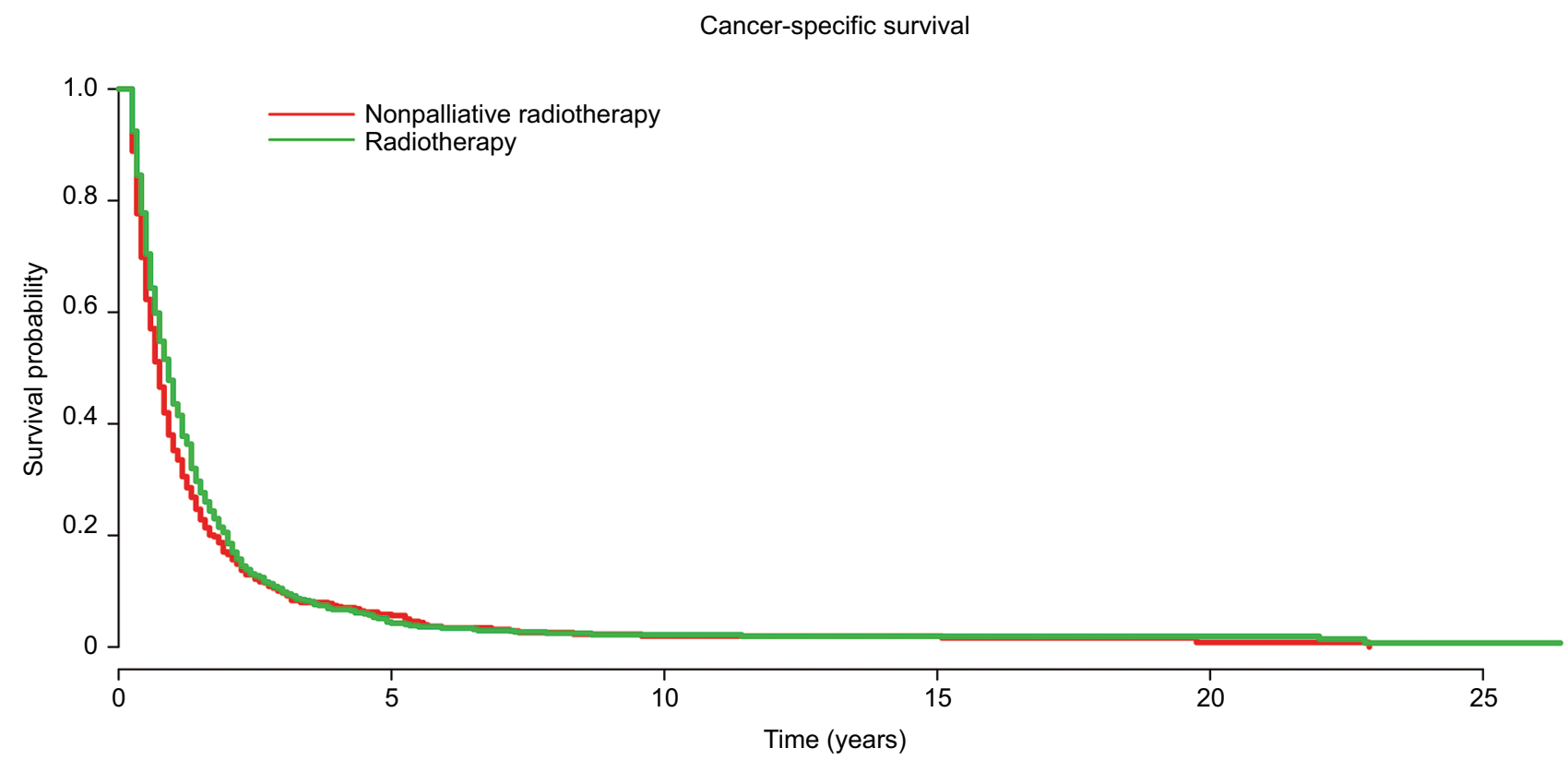

Figure 4 Kaplan-Meier analysis of (A) overall and (B) cancer-specific survival of the palliative radiotherapy and nonpalliative radiotherapy groups after propensity score matching.

\section{Disclosure}

The authors report no conflicts of interest in this work.

\section{References}

1. Patel T. Increasing incidence and mortality of primary intrahepatic cholangiocarcinoma in the United States. Hepatology. 2001;33(6):v1353-1357.

2. Shaib YH, Davila JA, McGlynn K, El-Serag HB. Rising incidence of intrahepatic cholangiocarcinoma in the United States: a true increase? J Hepatol. 2004;40(3):472-477.

3. McGlynn KA, Tarone RE, El-Serag HB. A comparison of trends in the incidence of hepatocellular carcinoma and intrahepatic cholangiocarcinoma in the United States. Cancer Epidemiol Biomarkers Prev. 2006;15(6):1198-1203.
4. Endo I, Gonen M, Yopp AC, et al. Intrahepatic cholangiocarcinoma: rising frequency, improved survival, and determinants of outcome after resection. Ann Surg. 2008;248(1):84-96.

5. Chou FF, Sheen-Chen SM, Chen YS, et al. Surgical treatment of cholangiocarcinoma. Hepatogastroenterology. 1997;44:760-765.

6. Shinohara ET, Mitra N, Guo M, Metz JM. Radiation therapy is associated with improved survival in the adjuvant and definitive treatment of intrahepatic cholangiocarcinoma. Int J Radiat Oncol Biol Phys. 2008;72:1495-1501.

7. Valle J, Wasan H, Palmer DH, et al. Cisplatin plus gemcitabine versus gemcitabine for biliary tract cancer. N Engl J Med. 2010;362:1273-1281.

8. Tao R, Krishnan S, Bhosale PR, et al. Ablative radiotherapy doses lead to a substantial prolongation of survival in patients with inoperable intrahepatic cholangiocarcinoma: a retrospective dose response analysis. J Clin Oncol. 2016;34(3):219-226. 
9. Chen YX, Zeng ZC, Tang ZY, et al. Determining the role of external beam radiotherapy in unresectable intrahepatic cholangiocarcinoma: a retrospective analysis of 84 patients. BMC Cancer. 2010; 10:492.

10. Jackson MW, Amini A, Jones BL, Rusthoven CG, Schefter TE, Goodman KA. Treatment selection and survival outcomes with and without radiation for unresectable, localized intrahepatic cholangiocarcinoma. Cancer J. 2016;22(4):237-242.

11. Amini N, Ejaz A, Spolverato G, Kim Y, Herman JM, Pawlik TM. Temporal trends in liver-directed therapy of patients with intrahepatic cholangiocarcinoma in the United States: a population-based analysis. J Surg Oncol. 2014;110(2):163-170.

12. Fritz A, Percy C, Jack A, et al. International Classification of Diseases for Oncology. Geneva, Switzerland: World Health Organization; 2000.

13. Joffe MM, Rosenbaum PR. Invited commentary: propensity scores. $A m$ J Epidemiol. 1999;150(4):327-333.

14. Austin PC. An introduction to propensity score methods for reducing the effects of confounding in observational studies. Multivariate Behav Res. 2011;46(3):399-424.

15. Ishwaran H, Kogalur UB, Blackstone EH, Lauer MS. Random survival forests. Ann Appl Stat. 2008;2(3):841-860.
16. Ho DE, Imai K, King G, Stuart EA. Matching as nonparametric preprocessing for reducing model dependence in parametric causal inference. Polit Anal. 2007;15:199-236.

17. van der Velden J, van Lindert AC, Gimbrere CH, Oosting H, Heintz AP. Epidemiologic data on vulvar cancer: comparison of hospital with population-based data. Gynecol Oncol. 1996;62(3):379-383.

18. Goodman KA, Wiegner EA, Maturen KE, et al. Dose-escalation study of single-fraction stereotactic body radiotherapy for liver malignancies. Int J Radiat Oncol Biol Phys. 2010;78(2):486-493.

19. Barney BM, Olivier KR, Miller RC, Haddock MG. Clinical outcomes and toxicity using stereotactic body radiotherapy (SBRT) for advanced cholangiocarcinoma. Radiat Oncol. 2012;7:67.

20. Tse RV, Hawkins M, Lockwood G, et al. Phase I study of individualized stereotactic body radiotherapy for hepatocellular carcinoma and intrahepatic cholangiocarcinoma. J Clin Oncol. 2008;26(4):657-664.

21. Mahadevan A, Dagoglu N, Mancias J, et al. Stereotactic body radiotherapy (SBRT) for intrahepatic and hilar cholangiocarcinoma. J Cancer. 2015;6(11):1099-1104.

22. Hong TS, Wo JY, Yeap BY, et al. Multi-institutional phase II study of highdose hypofractionated proton beam therapy in patients with localized, unresectable hepatocellular carcinoma and intrahepatic cholangiocarcinoma. J Clin Oncol. 2016;34(5):460-468.
Cancer Management and Research

\section{Publish your work in this journal}

Cancer Management and Research is an international, peer-reviewed open access journal focusing on cancer research and the optimal use of preventative and integrated treatment interventions to achieve improved outcomes, enhanced survival and quality of life for the cancer patient. The manuscript management system is completely online and includes

\section{Dovepress}

a very quick and fair peer-review system, which is all easy to use. Visit http://www.dovepress.com/testimonials.php to read real quotes from published authors. 